\title{
Radiomics in Oncology, Part 1: Technical Principles and Gastrointestinal Application in CT and MRI
}

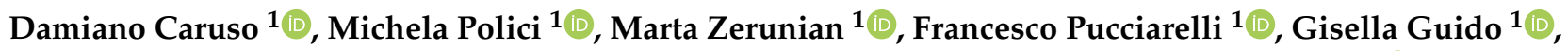 \\ Tiziano Polidori ${ }^{1}$, Federica Landolfi ${ }^{1}$, Matteo Nicolai ${ }^{1}$, Elena Lucertini ${ }^{1}$, Mariarita Tarallo ${ }^{2} \oplus$, Benedetta Bracci ${ }^{1}$, \\ Ilaria Nacci ${ }^{1}$, Carlotta Rucci ${ }^{1}$, Elsa Iannicelli ${ }^{1}$ and Andrea Laghi ${ }^{1, * \mathbb{D}}$ \\ 1 Radiology Unit, Department of Medical Surgical Sciences and Translational Medicine, Sapienza University \\ of Rome-Sant'Andrea University Hospital, Via di Grottarossa, 1035-1039, 00189 Rome, Italy; \\ damiano.caruso@uniroma1.it (D.C.); michela.polici@uniroma1.it (M.P.); \\ marta.zerunian@uniroma1.it (M.Z.); francesco.pucciarelli@uniroma1.it (F.P.); \\ gisella.guido@uniroma1.it (G.G.); tiziano.polidori@uniroma1.it (T.P.); federica.12005@libero.it (F.L.); \\ matteo.nicolai@uniroma1.it (M.N.); elena.lucertini@uniroma1.it (E.L.); benedetta.bracci@uniroma1.it (B.B.); \\ ilaria.nacci@uniroma1.it (I.N.); carlotta.rucci@uniroma1.it (C.R.); elsa.iannicelli@uniroma1.it (E.I.) \\ 2 Department of Surgery “Pietro Valdoni”, Sapienza University of Rome-Umberto I University Hospital, \\ Viale del Policlinico, 155, 00161 Rome, Italy; mariarita.tarallo@uniroma1.it \\ check for \\ * Correspondence: andrea.laghi@uniroma1.it; Tel.: +39-063-377-5285
} updates

Citation: Caruso, D.; Polici, M.; Zerunian, M.; Pucciarelli, F.; Guido, G.; Polidori, T.; Landolfi, F.; Nicolai, M.; Lucertini, E.; Tarallo, M.; et al. Radiomics in Oncology, Part 1: Technical Principles and Gastrointestinal Application in CT and MRI. Cancers 2021, 13, 2522. https: / / doi.org/10.3390/ cancers13112522

Academic Editors:

Antonia Dimitrakopoulou-Strauss, Khaled M. Elsayes and Tanya W. Moseley

Received: 31 March 2021

Accepted: 18 May 2021

Published: 21 May 2021

Publisher's Note: MDPI stays neutral with regard to jurisdictional claims in published maps and institutional affiliations.

Copyright: (c) 2021 by the authors. Licensee MDPI, Basel, Switzerland. This article is an open access article distributed under the terms and conditions of the Creative Commons Attribution (CC BY) license (https:/ / creativecommons.org/licenses/by/ $4.0 /)$.
Simple Summary: Part I is an overview aimed to investigate some technical principles and the main fields of radiomic application in gastrointestinal oncologic imaging (CT and MRI) with a focus on diagnosis, prediction prognosis, and assessment of response to therapy in gastrointestinal cancers, describing mostly the results for each pre-eminent tumor. In particular, this paper provides a general description of the main radiomic drawbacks and future challenges, which limit radiomic application in clinical setting as routine. Further investigations need to standardize and validate the Radiomics as a helpful tool in management of oncologic patients. In that context, Radiomics has been playing a relevant role and could be considered as a future imaging landscape.

Abstract: Radiomics has been playing a pivotal role in oncological translational imaging, particularly in cancer diagnosis, prediction prognosis, and therapy response assessment. Recently, promising results were achieved in management of cancer patients by extracting mineable high-dimensional data from medical images, supporting clinicians in decision-making process in the new era of target therapy and personalized medicine. Radiomics could provide quantitative data, extracted from medical images, that could reflect microenvironmental tumor heterogeneity, which might be a useful information for treatment tailoring. Thus, it could be helpful to overcome the main limitations of traditional tumor biopsy, often affected by bias in tumor sampling, lack of repeatability and possible procedure complications. This quantitative approach has been widely investigated as a non-invasive and an objective imaging biomarker in cancer patients; however, it is not applied as a clinical routine due to several limitations related to lack of standardization and validation of images acquisition protocols, features segmentation, extraction, processing, and data analysis. This field is in continuous evolution in each type of cancer, and results support the idea that in the future Radiomics might be a reliable application in oncologic imaging. The first part of this review aimed to describe some radiomic technical principles and clinical applications to gastrointestinal oncologic imaging (CT and MRI) with a focus on diagnosis, prediction prognosis, and assessment of response to therapy.

Keywords: Radiomics; oncologic imaging; Radiomics technical principles

\section{Introduction}

Radiomics is becoming central in emerging precision medicine, in particular for oncologic patients. Nowadays, conventional imaging is shifting from qualitative to quantitative 
approach, especially in tumor diagnosis, prognosis prediction, and assessment of response to therapy [1]. Radiomics has the expectancy to be an additional tool to provide a quantitative evaluation of tumor in a manner complementary to the observational approach and can be considered a helpful tool for physicians to manage oncologic patients by building a structured and objective workflow.

Radiomic parameters are ultrastructural quantitative data extracted from specific regions of interest (ROIs) [2] selected on encrypted medical images, that reflect neoplasm phenotypes and heterogeneity, usually correlated with tumor aggressiveness [2-4]. Then, radiologists, in a single image evaluation, can obtain an estimation of volumetric tumor heterogeneity in terms of radiomic parameters (i.e., tumor shape and textural parameters). In such scenario, oncologists may have an additional non-invasive biomarker to complement the biopsy that often can result too reductive or not diagnostic [5]. In addition, cancer biopsy could have several limitations related to risks for the patients (i.e., internal bleeding or pneumothorax), poor reproducibility, bias of random sample, limited accuracy in cancer grading and tumor invasion, and difficulty to sample some lesions [6,7]. Radiomics could be a supporting tool for cancer biopsy, a cornerstone of oncological management by guiding to the site with the most representative lesion heterogeneity, helpful for a better choice for biopsy or direct surgical resection rather than random sampling, and providing some additional information regarding tumor biology [8].

This recent landscape of imaging has been attracting the attention of many researchers, driven by promising results achieved through integration of Radiomics with clinical biomarkers. Song et al. reported that 553 original articles concerning Radiomics were published in the recent years [9]. The aim of this paper is to provide an overview of technical principles and the major radiomic studies that focused on diagnosis, prognosis assessment, and response to therapy in cancer patients, through an accurate description of prominent results in the most diffuse types of cancer with clinical significance. In Part 1, we will focus our attention on gastrointestinal applications.

\section{Technical Principles}

Radiomics workflow is divided into multi-steps: images acquisition, image segmentation, features extraction, features selection, model construction, and validation. Each step is dependent on the previous one and the aim is to obtain a performant and reliable prognostic model, usually driven by Artificial Intelligence, a research field in which the human intelligence is mimicked by mathematical and statistical approaches that are able to create performant artificial neural networks (Figure 1) [10]. Machine learning, neural network, and deep learning are some Artificial Intelligence subfields used for applying mathematical and statistical approaches to Big Data (e.g., radiomic features, clinical data, disease free survival, and overall survival) with the ability to find and interpret occult models, built on mineable data and their interpretation needed for clinical support [11]. To standardize radiomic approach in routine clinical setting, a structured and robust workflow is necessary, through which reliable and consistent data could be obtained.

The first step is based on images acquisition, which is one of the most challenging aspects because of the lack of protocol and parameters' standardization and the fact that it affects the reproducibility of analysis, particularly important in multicenter center studies [12]. Several options have been recently proposed to overcome the bias of acquisition protocols by performing a test-retest analysis, with the goal of eliminating radiomic features affected by higher variability and maintaining only robust features [13]. One of them is an automatic acquisition protocol that has been proposed among several CT scanners, and these methods showed some promising results in terms of reaching robustness in radiomic parameters by using resampling approach to uniform each voxel size as postacquisition correction. However, this approach has not been able to modulate acquisition specifications for each different CT scanner at the same time, and it could be difficult to use in retrospective studies routinely [14]. Regarding test-retest analysis, some relevant results have been obtained by analyzing the reproducibility of radiomic parameters among 
different $\mathrm{CT}$ scanners, both by changing $\mathrm{CT}$ specifications (intra-analysis) and by comparing different CT scanners (inter-analysis), and it was shown that high number of radiomic parameters could be altered by changing of some $\mathrm{CT}$ acquisition parameters [15]. However, these results were performed in phantom studies, in which the inter-patient variability has not been assessed; thus, future investigations are needed to identify the best approach to standardize the image acquisition. Nevertheless, the robust features often resulted to have low diagnostic performance [16]. In addition, applying a post-reconstruction batch harmonization has been also proposed to reduce the variability among centers by using global scaling, in which signal intensities are harmonized by eliminating the mean and the unit variance is downsized; z-standardization, where each feature is normalized considering the mean and standard deviation by providing some comparable results; and histogram-matching, by transforming intensity histogram in order to combine them and find the reference histogram [17]. However, each method has different advantages and drawbacks, and they should be weighted in different clinical scenarios.

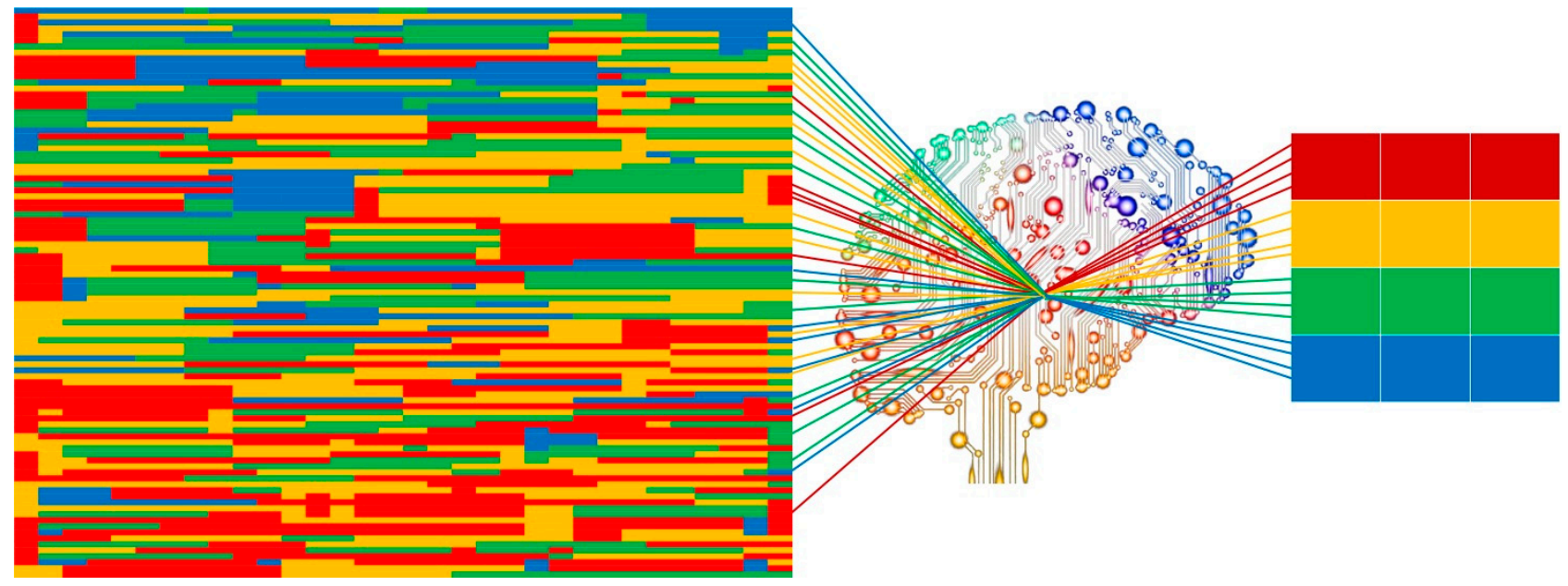

Figure 1. Graphic insight of Artificial Intelligence image analysis process. From imaging disorganized data to bright data.

The second phase is image segmentation, in which ROIs are outlined around cancer tissues, by covering the entire lesion area and avoiding some unnecessary structures (i.e., vessels, biliary duct, healthy parenchyma) that could alter the heterogeneity analysis. However, for very tiny non-lesion structures it is not always achievable, and this might represent a source of bias. The image segmentation is the leading cause of features variability, with a specific focus on inter-reader lack of reproducibility due to high variability of extracted features without a specific feature selection. High variability could affect the consistence of radiomic signature; thus, the reproducible features should be selected, excluding features classified as consistently unstable. Interclass correlation coefficient (ICC) might be used to evaluate the robustness of radiomic features among several datasets; in fact, there is evidence that a radiomic feature with high ICC on one dataset will also be robust and stable in other dataset $[18,19]$. Radiomic parameters are extracted from image volumes by performing manually, semi-automatic, or completely automatic segmentation. The first method is a time-consuming process, usually affected by high intra- and inter-observer variability and also according to radiologist's experience, and is difficult to apply in a clinical setting as a routine practice [20]. Semi-automatic and automatic segmentation process are demonstrated to be promising in homogeneous lesions, where interaction of external reader was minimum, with high accuracy, low inter-reader variability, and high reproducibility [21]. Since radiomic analysis could be highly affected by these different methods of segmentation, validation, standardization, and robustness are needed [22-24]. Consequently, the assessment of radiomic features variability related to segmentation processes has been emerging with the proposal to eliminate the features with high variability and low prognostic strength. 
Feature extraction represents the third step of radiomic process. Quantitative features have to be extracted from ROIs previously outlined on tissues of interest (Figure 2). Features obtained are divided into shape features, describing the shape and geometry of ROIs (i.e., volume, maximum surface): first-order statistics features, resulting from gray-level histogram and describing voxel values without considering the relationship with other voxels; second-order statistics features (i.e., gray-level co-occurrence matrices or graylevel run length matrices), derived by analyzing each pixel and its relationship with those adjacent in specific matrices; and high-order statistics features, resulting from mathematical algorithm after the application of specific filters (Table 1).To avoid some unnecessary bias, affecting data homogeneity, it is critical to standardize each step of analysis [25].

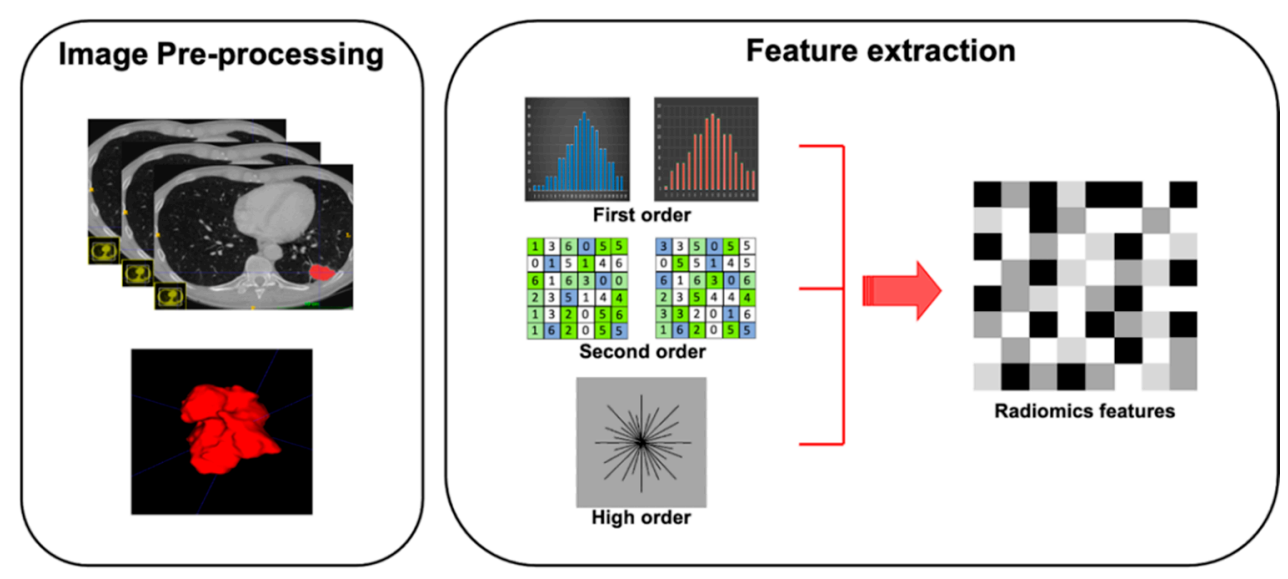

Figure 2. Graphical radiomic flowchart of features extraction. First order: description of voxel values without consider the relationship with others; second order: analysis of each pixel and its relationship with the adjacent pixels; higher order: result of mathematical algorithm performed with specific filters.

Table 1. Range of the first, second, and higher order textural features.

\begin{tabular}{|c|c|c|c|c|}
\hline Order & Features & Examples & Description & Comments \\
\hline First & $\begin{array}{c}\text { Pixel/Voxel Intensity } \\
\text { Histogram }\end{array}$ & $\begin{array}{l}\text { Kurtosis, skewness, first-order } \\
\text { entropy, mean of all pixels, mean } \\
\text { of positive pixels, } \\
\text { standard deviation }\end{array}$ & $\begin{array}{l}\text { Gray-level histogram, in } \\
\text { which x-axis represents } \\
\text { gray level of pixel/voxel } \\
\text { and y-axis the frequency } \\
\text { of occurrence }\end{array}$ & $\begin{array}{l}\text { Assessment of } \\
\text { pixel/voxel intensity } \\
\text { without consideration to } \\
\text { the relationship with } \\
\text { other pixels/voxels }\end{array}$ \\
\hline \multirow{2}{*}{ Second } & Run-length matrix & $\begin{array}{l}\text { Gray-level nonuniformity, } \\
\text { run-lenght nonuniformity, } \\
\text { long-run emphasis, } \\
\text { short-run emphasis }\end{array}$ & $\begin{array}{l}\text { Consecutive pixels/voxels } \\
\text { with the same gray level } \\
\text { and with a fixed direction }\end{array}$ & $\begin{array}{l}\text { Consider each } \\
\text { pixel/voxel intensity } \\
\text { and spatial relationships } \\
\text { with those adjacent }\end{array}$ \\
\hline & $\begin{array}{c}\text { Grey-level } \\
\text { co-occurence matrix }\end{array}$ & $\begin{array}{l}\text { Second-order entropy, sum of } \\
\text { entropy, sum of variance, } \\
\text { sum of averages }\end{array}$ & $\begin{array}{l}\text { How often occur in an } \\
\text { image pairs of pixels with } \\
\text { a specified spatial range } \\
\text { and specific value }\end{array}$ & \\
\hline Higher & Advanced metrics & $\begin{array}{c}\text { Geometry parameters, } \\
\text { neighborhood gray-tone difference } \\
\text { matrix, wavelet energy }\end{array}$ & $\begin{array}{l}\text { Relationships and } \\
\text { differences of multiple } \\
\text { pixels are compared }\end{array}$ & \\
\hline
\end{tabular}

Feature selection is one of the main key steps of radiomic process. It is related to the selection of best performing parameters among the large quantity of parameters extracted, often interconnected and characterized by overfitting. In that context, a specific and punctual selection of obtained features seems to be necessary to avoid some bias in model construction; it is essential to define the endpoint of analysis with precise clinical applications in order to select the best performing features with some dedicated approaches. Two most unsupervised approaches that are used to perform features selection are cluster 
analysis and principal component analysis. The first is based on clustering similar radiomic parameters according to high cluster redundancy and low inter-cluster correlation, usually illustrating as cluster heat map; and only one parameter from each cluster is selected for further analysis. The principal component analysis is built on creating a small set of non-correlated features extracted from a large amount of correlated features with the aim to explain the total variable variation using the smallest number of features, usually illustrating as score-plots [1]. The goal is to select the best features assessed as nonredundant, reproducible, and performing.

The next step is the model building in which the best selected features, clinical data, and histological data are combined to assess the pre-fixed outcome (e.g., survival, disease free progression, and therapeutic assessment). In this critical step, several approaches have been proposed to perform multivariate analysis, tailored per endpoint, which could provide a clinical tool able to support clinical decision-making. Different statistical methods and data mining and/or machine-learning methods were investigated. The main classifiers used in practice are random forest, linear regression, logistic regression, Cox proportional hazards regression, least absolute shrinkage and selection operator (LASSO), support vector machines (SVM), neural network, deep learning, and decision tree [26]. To date, it was shown that there is no unique best classifier, but each of them should be evaluated in order to obtain the consistent, reliable, and generalizable models [27].

In conclusion, the constructed model must be trained and optimized on training and testing set, then validated on external cohort in order to obtain a reliable model on different patient groups. Although the validation on external datasets is necessary, sometimes it could be impossible. Hence, several strategies have been tested to overcome this limitation by obtaining an internal validation (i.e., random subsampling and nested approach) but the main issue of these approaches is the risk to alter the features selection algorithm, and the results obtained could be unreasonably optimistic [28]. To sum up, the validation model achieved through external validation is the goal standard and the comparability of radiomic features extracted from medical images, acquired with different protocols, and segmentation processes performed with different tasks are the main challenges of radiomic approaches.

\section{Esophageal Cancer}

Several studies investigated Radiomics in staging, response to therapy prediction, and post-operative recurrence of esophageal squamous cell carcinoma (ESCC). Regarding ESCC staging, Wu et al. [29] proposed a radiomic approach, based on computed tomography (CT) late arterial phase, by performing manual segmentation. Ten radiomic features were selected to build the signature, and the radiomic model resulted to be superior to tumor volume, a morphological characteristic reflecting tumor size, in distinguishing early (I-II) from advanced stage (III-IV), both in primary (AUC 0.795 vs. 0.694 , respectively) and validation cohort (AUC 0.762 vs. 0.624 , respectively). Radiomic approach was also confirmed by Liu and colleagues [30], who proposed CT Texture Analysis (CTTA) on baseline CT as a possible biomarker to evaluate ESCC aggressiveness (T, N, overall stages, and grading differentiation) in the preoperative clinical setting. Kurtosis and entropy were found as independent predictors for T stages (T1-2 vs. T3-4) and overall stages (I-II vs. III-IV); in addition, entropy showed the best performance in terms of T stages (T1-2 vs. T3-4), lymph node metastasis ( $\mathrm{N}-$ vs. $\mathrm{N}+$ ), and overall stages (I/II vs. III/IV) with AUC $0.637,0.815$, and 0.778 , respectively.

Radiomics also resulted to be promising in the pretherapeutic assessment of chemotherapy response [31-33]. Jin et al. [31] tested an integrated model of CT radiomic features using machine learning approach in 94 patients affected by esophageal cancer who underwent neoadjuvant chemotherapy (CRT) (58 responders and 36 non-responders). A total of 42 radiomic features were extracted, and the integrated radiomic and dosimetric model obtained a good accuracy (AUC 0.708) in predicting the treatment response of CRT in patients with ESCC. Differently, Nakajo and colleagues [33] investigated the heterogene- 
ity of F-18-fluorodeoxyglucose (18F-FDG) distribution in predicting tumor response in 52 patients affected by esophageal cancer. Despite textural parameters and volumetric features correlating with tumor response, texture analysis did not show a relevant role in estimation of esophageal cancer prognosis. Among treatment assessment, $\mathrm{Hu}$ and colleagues [32] tested Radiomics to evaluate tumor complete response in 231 patients with locally advanced ESCC treated with neoadjuvant CRT, by combining intratumoral and peritumoral features, obtaining an AUC of 0.852.

Furthermore, several studies focused on predicting risk of pathological lymph nodes (LN) in esophageal cancer patients such as that of Wu et al. [34] and Shen et al. [35] in which the authors proposed Radiomics as a tool in pretherapeutic LN assessment. A Radiomic signature was built from 13 radiomic features extracted from 197 ESCC patients, and it was significantly associated with pathological LN $(p<0.001)$. Moreover, the AUC was 0.806 in the training model and 0.771 in the validation model, suggesting that Radiomics may be helpful for suspected LN.

Recently, radiomic application in recurrency has been investigated by Qiu et al. [36] validating a radiomic combined nomogram (quantitative and clinical parameters) based on preoperative CT scans of 206 ESCC patients treated with neoadjuvant CRT and surgery; the model showed high correlation with recurrence-free survival both in training (AUC 0.746 ) and validation cohort (AUC 0.724).

\section{Gastric Cancer}

In the last few years, several authors have also investigated Radiomics in gastric cancer (GC) management, and the major results were obtained in diagnosis and staging, prognosis stratification, and treatment response prediction. In a preoperative clinical setting, Radiomics was applied to evaluate histopathological tumor characteristics, and to predict risk of nodal and peritoneal metastases [37-39]. Liu and colleagues studied CTTA to evaluate histopathological findings in 392 pre-operative CT scans by using endoscopic biopsy as the reference standard [37]. CTTA was performed on arterial and venous phase, and each ROI was manually drawn on selected slice where cancer area was depicted. Authors made a comparison between CTTA features and histological data. They obtained that mean attenuation, maximum attenuation, all percentiles, and mode extracted from CT venous phase correlated with tumor differentiation degree (from $\mathrm{r}=-0.231$ to $\mathrm{r}=-0.324, p<0.017$ ), and hence CTTA could be helpful to identify well differentiated cancers $(p<0.004)$. This radiomic approach was reinforced by Wang et al. [38], who obtained some relevant results by testing a CT-based radiomic nomogram (radiomic features + clinical parameters) to predict pathological LN before any therapy. By using a random forest algorithm, radiomic model showed a good power in discriminating pathological from non-pathological LN with an AUC of 0.844 and 0.837 for training and test population, respectively, while the radiomic nomogram showed better results for both training and test cohort with an AUC of 0.886 and 0.881 , respectively.

Surprising results were obtained by Dong and colleagues [39] aimed at using a radiomic approach in identifying occult peritoneal metastases (PM) in a multicentric study listing 554 patients affected by gastric cancer in pretherapeutic setting. All patients have a negative CT for peritoneal involvement while laparoscopy confirmed the presence of PM in $122 / 554$ patients. In total, 266 quantitative radiomic features were extracted from primary tumor and from peritoneum region, and the predicting power of the derived radiomic nomogram was surprising with an AUC of $0.958,0.928$, and 0.920 for training and two external validation datasets, respectively.

Recently, Giganti et al. [40] proposed CTTA to estimate pretherapeutic overall survival (OS) in 56 patients with resectable GC by performing a manually volumetric segmentation of entire tumor volume. Authors revealed that some textural parameters of heterogeneity, extracted from preoperative CT scans, were strictly linked to poor prognosis $(p<0.046)$. Furthermore, Li et al. [41] confirmed these promising results of Radiomics in GC prediction of prognosis by analyzing 181 GC patients who underwent radical resection and then 
analyzing radiomic signature alone and combining clinical-pathological data. Their results revealed that radiomic integrated nomogram had a higher prognostic value in stratifying patients into high- and low-risk of death (Harrel concordance index, 0.82 vs. $0.74, p<0.001$ ).

Only few studies investigated Radiomics in pretherapeutic prediction of chemotherapy response. Jiang et al. [42] developed a radiomic score to evaluate stage II and III in patients that could benefit from adjuvant chemotherapy. They divided patients into low, medium or high radiomic score categories based on 1147 baseline $\mathrm{CT}$, and a comparison was made between disease-free survival (DFS) and OS of patients who received chemotherapy and patients who did not. Results showed that the advantage of adjuvant chemotherapy for patients with high radiomic score was higher $(\mathrm{HR}<0.412, p<0.001)$ than patients with low or medium score. Similar and concordant results were obtained by Sun et al. [43], which validated radiomic and clinical scores based on 106 CT (74 training and 32 validation cohort) of patients at III and IV clinical stage in order to predict neoadjuvant chemotherapy (NAC) response. They showed higher performance of Radiomics (AUC 0.77 training cohort; AUC 0.82 validation cohort) in comparison with only clinical score (AUC 0.70 training cohort; AUC 0.62 validation cohort); however, they did not obtain statistically significant differences between two scores $(p=0.15)$.

\section{Liver and Biliary Tract}

The liver cancer workup has been extensively debating, and hepatocellular carcinoma (HCC) diagnosis represents the challenge of imaging in cirrhotic patients having several indeterminate nodules. In this context, Radiomics was recently proposed as an additional tool to identify HCC nodule and assess HCC grading [44,45]. Mokrane et al. [44] extracted CT quantitative parameters from 178 cirrhotic patients in order to identify HCC among indeterminate nodules, in the light of optimizing patient management and improving outcome by reducing the rate of liver biopsy or wait-and-see strategy in fragile patients. No significant results were reported by comparing HCC and non-HCC nodules using LI-RADS scores, while radiomic signature, based on delta quantitative features (between venous and arterial phases), reached an AUC of 0.70 in training cohort (142 patients) and 0.66 in validation cohort (36 patients).

$\mathrm{Wu}$ and colleagues [45] aimed to investigate magnetic resonance imaging (MRI) radiomic signature to distinguish low- from high-grade HCC. The authors showed relevant results to differentiate two patient groups $(p<0.05)$, combining radiomic and clinical features, with an AUC of 0.800 . Furthermore, both radiomic signature and alpha fetoprotein level resulted to be factors independent of HCC grading $(p<0.05)$.

Regarding cholangiocarcinoma diagnosis, Yang et al. [46] retrospectively performed a radiomic analysis on preoperative MRI images in 100 patients with pathological diagnosis of extrahepatic cholangiocarcinoma aiming at detecting both differentiation degree (highand medium-low differentiation) and nodal status in pretherapeutic clinical setting. A radiomic model was built incorporating radiomic features and ADC value obtaining significant results in differentiation degree (AUC 0.78) and in nodal status prediction (AUC 0.80).

Regarding OS, Kim et al. [47] proposed three scores (radiomic, clinical alone, and combined) to assess OS in patients affected by HCC and treated with trans-arterial chemoembolization showing the combined score as the better survival predictor with a HR of $19.88(p<0.0001)$. Microvascular invasion (MVI) was shown to be one of the main predictors of outcome and tumor grading in HCC patients, and more aggressive surgery could be planned in case of MVI prediction. To date, preoperative detection still represents an imaging challenge compared with histology as a reference standard. Recently, high interobserver variability has also been shown in MVI evaluation on MRI among expert radiologists, showing poor performance [48]. In such scenario, Radiomics could be a key tool as shown by $\mathrm{Xu}$ et al. [49], who created and tested a radiographic-radiomic, CT-based model, including clinical, radiomic, and radiographic parameters, and obtained a high precision in predicting pretherapeutic MVI (AUC 0.88). Ni and colleagues [50] explored 
a performant diagnostic model to predict MVI on CT scans, obtaining substantial results in agreement with a previous study. In total, 1044 textural features were extracted from CT in 88 MVI-positive and 118 MVI-negative patients. Twenty-one radiomic methods were tested, showing promising results for the LASSO method combined with gradient boosting decision tree which reached the best performance in distinguishing MVI-positive and MVI-negative (accuracy $84 \%$, sensitivity $82 \%$, specificity $85 \%$, and AUC 0.88 ). Among MRI, Feng et al. [51] developed and validated a radiomic model in 110 HCC patients before surgery; intratumoral and peritumoral radiomic features were extracted, and the resulting model demonstrated good performance in MVI prediction with AUC of 0.85 both in training and validation cohorts.

Radiomics was also extensively studied to predict the risk of recurrence, both in HCC and in cholangiocarcinoma [52,53]. Radiomic and machine learning approach were extensively investigated by Ji et al. [52] to predict HCC recurrence in 470 patients, who underwent pretherapeutic $\mathrm{CT}$ and surgical resection of solitary nodule. The authors developed and validated two models, combining radiomic and clinical factors, that were able to predict risk of recurrence pre- and post-surgery. A three-feature signature was developed obtaining favorable results in predicting HCC recurrence (C-index of 0.699). Similarly, Liang et al. [53] conducted a single-center retrospective study developing a radiomic nomogram, based on pretherapeutic arterial-phase contrast-enhanced MRI, in order to predict early recurrence of intrahepatic cholangiocarcinoma after primary surgery. Radiomic combined model reached a better performance to predict risk of recurrence after surgery with AUC of 0.9 and 0.77 in training and validation cohorts, respectively.

In conclusion, liver metastases was an interesting research field in colorectal cancer, where texture features have shown a remarkable value as imaging biomarker, capable of differentiating patients with high- and low-risk of developing synchronous or metachronous liver metastases [54]. Taghavi et al. [54] concluded that a hybrid model, combining clinical and texture parameters, achieved the best prediction performance, yielding an AUC of $86 \%$ in predicting the occurrence of liver metastases and demonstrating that a non-invasive, artificial-intelligence-based model could support individualized therapy and improve oncological outcome.

\section{Pancreas}

Application of Radiomics in pancreatic cancer was mostly studied to diagnose pancreatic ductal adenocarcinoma (PDAC), to assess response to therapy, and to predict overall survival (Figure 3). In particular, Radiomics was investigated to overcome CT scans' limitations in differential diagnosis between PDAC and pancreatitis, which could often mimic cancer [55-58]. Ren et al. [55] applied Radiomics on CT to differentiate PDAC from massforming pancreatitis. The authors retrospectively enrolled 109 patients (79 with PDCA and 30 with mass-forming pancreatitis) who underwent unenhanced CT prior to surgery. Volumetric Radiomics was extracted from CT images and then diagnostic performance was assessed considering histology as a reference standard. Among 396 radiomic features extracted, the four most significant predictive parameters were considered to build a predictive model with a high accuracy, sensitivity, and specificity $(93.3 \%, 92.2 \%$, and $94.2 \%$, respectively). Since then, other differential diagnosis evaluated with Radiomics included autoimmune pancreatitis and healthy pancreatic parenchyma. In fact, Park and colleagues [57], reinforcing the previous study, performed a CT-machine learning method to differentiate autoimmune pancreatitis from PDAC. Autoimmune pancreatitis is an entity pathologically distant from PDAC but visual imaging features have an important overlap, making difficult both the discrimination between the two and the consideration of different treatment options. Two types of CT Radiomics were extracted based on different CT phases (arterial and portal-venous phases) and slice thickness (thin and thick slices). The study population was divided into test and validation cohorts and a random forest classifier was applied to select the most significant features. CT Radiomics extracted from thin slice portal-venous phase showed the correct classification of 100\% (33/33) of the PDAC 
included in the validation cohort, with sensitivity, specificity and accuracy of $89.7 \%, 100 \%$, and $95.2 \%$, respectively. On the other hand, Chu et al. [56] tested the Radiomics' ability to identify PDAC from normal pancreatic tissue, performing 3D manual segmentation of the whole pancreatic parenchyma, assessed on portal-venous phase. One-hundred and ninety PDAC and 190 healthy patients were analyzed, and Radiomics yielded an accuracy of $99.2 \%$ (AUC 0.99 , sensibility 100\%, and specificity $98.5 \%$ ) in the diagnosis of PDAC cases.

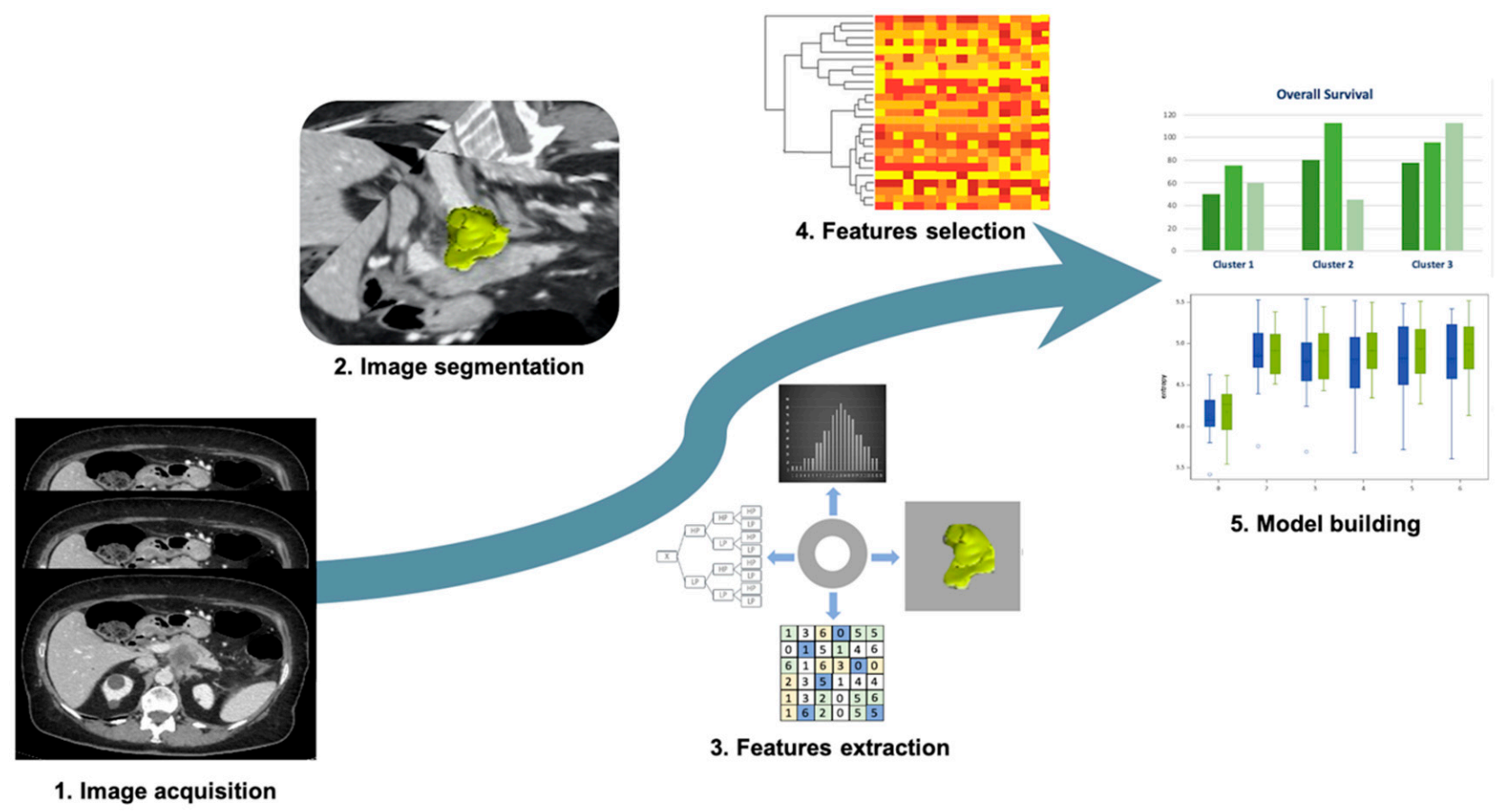

Figure 3. Radiomic approach on pancreatic ductal adenocarcinoma. 1. Image acquisition: CT scans acquired on venous phase; 2. Image segmentation: semiautomatic segmentation performed on CT scans; 3 . Feature extraction: tumor shape, first order features, texture features, and wavelet filter; 4 . Feature selection: cluster analysis was used for the features selection; 5. Model building: survival and clinical data combined with radiomic features.

Radiomics was also tested in the evaluation of pretherapeutic response to CRT by Nasief et al. [59], who investigated the predictive value of delta-Radiomics on 28 unenhanced CT scans obtained during therapy. Good performance resulted in distinguishing good from bad responders (AUC 0.94). Similar results were obtained by Parr et al. [60], who developed a CT-based radiomic model to assess the clinical outcome in 74 patients who underwent stereotactic body radiotherapy. The radiomic-model achieved a better performance (AUC 0.78) than clinical model (AUC 0.66) in differentiating high- from low-risk patients.

Furthermore, several studies investigated Radiomics as non-invasive biomarker in prognosis and OS prediction in PDAC. Eilaghi et al. [61] applied CT texture analysis (CTTA) on patients with resectable PDAC to assess OS and achieved a statistically significant correlation $(p<0.05$ and AUC 0.72) between OS and two textural parameters (dissimilarity and inverse difference normalized). Recently, Kim et al. [62] proposed to test CTTA to measure OS in PDAC patients, who underwent surgery after NAC. They calculated delta textural features between pre-surgery and baseline CT images and found a statistically significant correlation between CTTA features and OS (higher subtracted entropy, $p=0.005$ and $\mathrm{HR}=0.159$, and lower subtracted GLCM entropy, $p=0.036$ and HR $=10.325$ ). In addition, Tang et al. [63] developed a radiomic MRI-nomogram based on 303 patients with resectable PDAC to stratify early recurrence risk. Combining radiomic and clinical parameters, they showed an AUC between 0.88 and 0.85 depending on training and internal and external validation cohorts. 


\section{Small Bowel}

Small bowel neoplasms are rare entities, and early-stage small bowel tumors are difficult to recognize on conventional imaging. Even if esophagogastroduodenoscopy with endoscopic ultrasound is recommended during initial workup, CT or MRI may be useful to evaluate the extent of local tumor invasion and to evaluate distant metastases [64].

Currently, radiomic-based models that are able to diagnose and characterize small bowel tumors are a few in number, mostly dedicated to a specific histotype of small bowel tumors. In particular, Lu and colleagues [65] performed a first-order histogram-based analysis on 74 patients to differentiate duodenal adenocarcinoma (DAC), PDAC, and gastrointestinal stromal tumor (GIST) of periampullary region. Whole-lesion CT histogram analysis demonstrated significant differences among DAC, PDAC, and GIST (all $p<0.05$ ), whereas performance analysis reported significant AUC for the 90th percentile on portalvenous phase for differentiating DAC from PDAC (AUC 0.854, sensitivity 92.3\%, and specificity $80 \%$ ), while the 90th percentile on arterial phase was the most accurate in the differentiation between DAC and GIST (AUC 0.809, sensitivity $100 \%$, and specificity $64 \%$ ) and between PDAC and GIST (AUC 0.936 sensitivity $90 \%$, and specificity $86 \%$ ).

Interesting studies on GIST have recently been performed for risk stratification on baseline CT [66-68], about DFS and disease progression after surgery [69] or after therapy with tyrosine kinase inhibitors [70]. For example, Ren [66] and colleagues validated a nomogram based on Radiomics and CT signs together, to preoperatively predict malignancy in 440 GIST patients. Interesting results in differentiating low-malignant from high-malignant GIST were reported by them, and the radiomic nomogram was identified as an excellent discriminator with AUC of 0.935 and 0.933 for training and validation datasets, respectively. These encouraging results might be useful in the future to build robust decision models.

\section{Neuroendocrine Tumors}

Radiomics was recently applied to manage neuroendocrine tumors (NETs), extremely rare tumors. Few studies proposed a Radiomics approach in pancreatic neuroendocrine tumors (PNETs) to assess histological degree, differential diagnosis, and prognosis. In particular, Guo et al. [71] tested CTTA ability to differentiate PNETs G1/G2 from G3, by performing a manual 2D primary lesions' segmentation on baseline contrast-enhanced CT. Some textural parameters differ between two patient groups with good sensitivity (73-91\%) and specificity (85-100\%). Similar and concordant results were proposed by Gu and colleagues [72], who performed a CT-radiomic nomogram, combining quantitative and clinical factors, to predict PNETs grading (G1 and G2/G3) on 138 patients, who underwent contrast-enhanced CT at diagnosis. They demonstrated that the combined model was extremely promising to differentiate PNET G1 from G2/G3 with AUC 0.97 in training and 0.90 in validation cohort. In addition, Bian and colleagues [73] performed a radiomic model to evaluate preoperative tumor grading in patients affected by non-functional PNETs on 3T MRI and confirmed promising radiomic results. They visually evaluated non-functional PNETs for radiological findings used for the clinical score and then selected a volume of interest (VOI) of the lesion to extract radiomic features. Both clinical model and radiomic model were obtained; results were obtained on 97 patients and then validated on 42 patients. An AUC of 0.769 and 0.72 (training and validation group, respectively) was reached by the clinical model including 14 imaging features, while 14 radiomic features showed good discrimination in the training cohort (AUC 0.851) and the validation cohort (AUC 0.736).

He and colleagues [74] built and validated a CT-based radiomic model to differentiate atypical non-functional PNETs from PDAC, by testing radiomic features alone and in combination with clinic-radiological features. Radiomics was extracted from preoperative enhanced CT of 147 patients. Three models (only clinical-radiological, only Radiomics, and combined) were trained and validated to differentiate atypical non-functional PNET from PDAC. The clinical-radiological model showed an AUC of 0.775 , while the radiomic model 
reached an AUC of 0.873 . The combined model resulted in a slightly higher performance than radiomic model, with an AUC of 0.884 .

Finally, regarding differential diagnosis among NETs, Martini et al. [75] evaluated ability of CTTA, extracted from liver metastases, to differentiate pancreatic and non-pancreatic NETs with the same grading (G1 or G2). Volumetric analysis on liver metastasis was performed on 48 patients ( 23 with PNET and 25 with non-pancreatic NET). Their results showed how first-order Radiomics provided some relevant differences in differentiating PNETs from non-pancreatic NETs in both arterial phase (skewness feature, G2) and portal-venous phase (mean feature, for G1 and G2), all $p<0.05$.

In addition, interesting correlation was also found regarding Radiomics with OS (mean feature extracted from both arterial and portal-venous phase, $r>0.42$ ) and with time to progression (entropy feature extracted from arterial phase, $\mathrm{r}<-0.42$ ).

No differences emerged to discriminate tumor lesions for grading (G1 vs. G2); as well as no significant correlation were observed between Radiomics and Ki-67 in both PNETs and non-pancreatic NETs groups.

\section{Colorectal Cancer}

Radiomics and Radiogenomics were also widely investigated as imaging biomarker in colorectal cancer (CRC) in the assessment of mutational status, nodal metastases, stratification patient risk, and in evaluation of response to therapy [76-81]. Regarding CRC Radiogenomics, Yang et al. [82] retrospectively investigated whether CT-based radiomic signature could predict KRAS, NRAS, and BRAF mutations in CRC by analyzing a primary cohort ( 61 patients) and a validation cohort (56 patients). They made a correlation between radiomic features, clinical data, and genetic mutations and reported a significant $(p<0.001)$ association between mutational status and the proposed radiomic signature (AUC 0.869 , sensitivity 0.857 , and specificity 0.833 ). No significant results were obtained in the comparison between clinical/histological data and genetic profiling $(p>0.05)$. In addition, multifunctional imaging has been tested for CRC genotyping; Taguchi et al. [83] compared the CTTA with Maximum Standard Uptake Values $\left(\mathrm{SUV}_{\max }\right)$ performance from ${ }^{18} \mathrm{~F}-\mathrm{FDG}$ PET/CT, and concluded that Radiomics was superior in distinguishing between wild type and mutant KRAS colon cancers (AUC 0.82 vs. 0.58) in agreement with the previous study. Golia Pernicka et al. [84] enhanced Radiogenomics results in prediction prognosis and developed some models based on clinical and radiomic parameters, and compared their performances in the assessment of microsatellite instability (MSI); the combined clinical-radiomic model achieved the best results in predicting MSI (AUC 0.79, specificity $92.5 \%$, sensitivity $31.6 \%$, positive predictive value (PPV) $66.7 \%$, and negative predictive value (NPV) $74 \%$ ).

Li et al. [85] tested Radiomics as a pretherapeutic tool in the investigation of pathological LN before starting any therapy. Six predictive models were compared, and the most relevant results were obtained by combining clinical variables and LN-radiomic features, extracted from primary tumor and peripheral nodes in 458 patients of primary cohort and 308 of validation cohort (AUC 0.75 , accuracy $73 \%$, sensitivity $60 \%$, and specificity $84 \%$ in testing set). Regarding rectal cancer, in order to assess pretherapeutic nodal status by employing textural features extracted from primary tumor on baseline MRI scans, Radiomics has been reported to range from $50 \%$ to $93 \%$ for sensitivity and from $43 \%$ to $80 \%$ for specificity in the latest studies $[86,87]$ with a good interobserver agreement reproducibility in clinical practice, confirming the relevant results previously described.

Recently, a radiomic classifier has been tested to assess the response to treatment demonstrating a better performance compared with traditional evaluation (T2w and DWI), showing an AUC of $93 \%$ in distinguishing complete from partial responder patients, affected by rectal cancer, showing sensitivity, specificity, PPV, and NPV of 100\%, 91\%, $72 \%$, and $100 \%$ respectively, (vs. $84 \%, 56 \%, 94 \%$, and $30 \%$ achieved with T2w-DWI qualitative assessment) [88]. 


\section{Limitations GI Radiomics}

To date, Radiomics has several relevant drawbacks that limit the routine use in the clinical management of gastrointestinal cancer patients. The major radiomic limitations are the lack of standardization, both in image protocols of acquisition and in methods of segmentations; the lack of validation in prospective and large sample of patients, enrolled from multicentric studies; and an undefined correlation with histological diagnosis. These aspects could affect the reliability and stability of radiomic features, especially when extracted from small sample of patients, in which the risk of oversampling has to be considered. In particular, the application of Radiomics in differential diagnosis between benign and malignant lesions needs to be validated and compared with histological results, considered as a reference standard. In addition, nodal metastases and genetic panel prediction has to be further validated in larger patient populations. Nevertheless, the application of Radiomics has been shown to be a promising tool in each step of cancer patient's workup, able to support clinical approach to tailor treatment per patients. In the future, Radiomics should be investigated and validated in a more structured manner to overcome the major limitations.

\section{Conclusions}

Up to now, Radiomics is an emerging and promising imaging tool for oncologist in the development of personalized cancer medicine. Quantitative approach in medical imaging has been overcoming the traditional subjective evaluation, resulting to be more promising in the assessment of tumor aggressiveness and patient's survival. Radiomics has been extensively studied in the management of cancer patients as a complementary tool for the clinicians, and the major results were obtained by combining clinical and quantitative data. In Part I, the main radiomic technical challenges were the lack of standardization in images acquisition, segmentation, extraction, and processing features. Actually, Radiomics resulted to be absolutely promising in diagnosis, prediction prognosis, overall survival, and risk of recurrence, representing the future application of radiology. However, it will be necessary to investigate the feasible use of Radiomics in clinical practice by providing structured models in gastrointestinal oncologic imaging to overcome the lack of repeatability and reproducibility.

Author Contributions: Conceptualization, D.C., M.P. and A.L.; methodology, D.C., M.P., M.Z.; validation, A.L. and M.Z.; formal analysis, G.G., T.P., C.R.; data curation, M.P., M.Z., C.R. and F.L.; writing—original draft preparation, F.P., E.L., T.P., G.G., M.N., F.L., B.B., I.N.; writing—review and editing, D.C., M.P., M.Z. and E.I.; visualization, D.C., M.T. and A.L.; supervision, A.L.; project administration, D.C. and A.L. All authors have read and agreed to the published version of the manuscript.

Funding: This study is funded by AIRC (Associazione Italiana per la Ricerca sul Cancro) IG 2020/24974.

Institutional Review Board Statement: The study was conducted according to the guidelines of the Declaration of Helsinki, and approved by the Institutional Review Board of Sant'Andrea Hospital, Rome, Italy.

Informed Consent Statement: Informed consent was obtained from all subjects involved in the study.

Conflicts of Interest: The authors declare no conflict of interest.

$\begin{array}{ll}\text { Abbreviations } \\ \text { MRI } & \begin{array}{l}\text { magnetic resonance imaging } \\ \text { computed tomography }\end{array} \\ \text { CT } & \text { positron emission tomography } \\ \text { PET } & \text { diffusion weighted imaging } \\ \text { DWI } & \text { apparent diffusion imaging } \\ \text { ADC } & \text { dynamic contrast enhanced } \\ \text { DCE } & \text { diffusion kurtosis imaging }\end{array}$




$\begin{array}{ll}\text { AUC } & \text { area under the curve } \\ \text { ROC } & \text { receiver operating characteristic } \\ \text { ICC } & \text { interclass correlation coefficient } \\ \text { SVM } & \text { support vector machine } \\ \text { CNN } & \text { convolutional neural networks } \\ \text { CTTA } & \text { CT texture analysis } \\ \text { PPV } & \text { positive predictive value } \\ \text { NPV } & \text { negative predictive value } \\ \text { OS } & \text { overall survival } \\ \text { DFS } & \text { disease-free survival } \\ \text { PFS } & \text { progression-free survival } \\ \text { CRT } & \text { chemoradiation therapy } \\ \text { SUV } & \text { standard uptake values } \\ \text { ESCC } & \text { esophageal squamous cell carcinoma } \\ \text { 18F-FDG } & \text { F-18-fluorodeoxyglucose } \\ \text { LN } & \text { lymph nodes } \\ \text { GC } & \text { gastric cancer } \\ \text { PM } & \text { peritoneal metastases } \\ \text { NAC } & \text { neoadjuvant chemotherapy } \\ \text { HCC } & \text { hepatocellular carcinoma } \\ \text { MVI } & \text { microvascular invasion } \\ \text { PDAC } & \text { pancreatic ductal adenocarcinoma } \\ \text { DAC } & \text { duodenal adenocarcinoma } \\ \text { GIST } & \text { gastrointestinal stromal tumor } \\ \text { NET } & \text { neuroendocrine tumors } \\ \text { PNET } & \text { pancreatic neuroendocrine tumors } \\ \text { CRC } & \text { colorectal cancer } \\ \text { MSI } & \text { microsatellite instability } \\ & \end{array}$

\section{References}

1. Rizzo, S.; Botta, F.; Raimondi, S.; Origgi, D.; Fanciullo, C.; Morganti, A.G.; Bellomi, M. Radiomics: The facts and the challenges of image analysis. Eur. Radiol. Exp. 2018, 2. [CrossRef] [PubMed]

2. Lambin, P.; Leijenaar, R.T.H.; Deist, T.M.; Peerlings, J.; de Jong, E.E.C.; van Timmeren, J.; Sanduleanu, S.; Larue, R.T.H.M.; Even, A.J.G.; Jochems, A.; et al. Radiomics: The bridge between medical imaging and personalized medicine. Nat. Rev. Clin. Oncol. 2017, 14, 749-762. [CrossRef]

3. Bodalal, Z.; Trebeschi, S.; Nguyen-Kim, T.D.L.; Schats, W.; Beets-Tan, R. Radiogenomics: Bridging imaging and genomics. Abdom. Radiol. 2019, 44, 1960-1984. [CrossRef] [PubMed]

4. Miles, K. Radiomics for personalised medicine: The long road ahead. Br. J. Cancer. 2020, 122, 929-930. [CrossRef]

5. Khan, T.; Usman, Y.; Abdo, T.; Chaudry, F.; Keddissi, J.I.; Youness, H.A. Diagnosis and management of peripheral lung nodule. Ann. Transl. Med. 2019, 7. [CrossRef]

6. $\quad$ Brennan, M.E.; Turner, R.M.; Ciatto, S.; Marinovich, M.L.; French, J.R.; Macaskill, P.; Houssami, N. Ductal carcinoma in situ at core-needle biopsy: Meta-analysis of underestimation and predictors of invasive breast cancer. Radiology 2011, 260, 119-128. [CrossRef] [PubMed]

7. Liu, L.; Yi, X.; Lu, C.; Qi, L.; Zhang, Y.; Li, M.; Xiao, Q.; Wang, C.; Zhang, L.; Pang, Y.; et al. Applications of radiomics in genitourinary tumors. Am. J. Cancer Res. 2020, 10, 2293-2308. [PubMed]

8. Gillies, R.J.; Kinahan, P.E.; Hricak, H. Radiomics: Images are more than pictures, they are data. Radiology 2016, $278,563-577$. [CrossRef] [PubMed]

9. Song, J.; Yin, Y.; Wang, H.; Chang, Z.; Liu, Z.; Cui, L. A review of original articles published in the emerging field of radiomics. Eur. J. Radiol. 2020, 127. [CrossRef]

10. Shimizu, H.; Nakayama, K.I. Artificial intelligence in oncology. Cancer Sci. 2020, 111, 1452-1460. [CrossRef]

11. Attanasio, S.; Forte, S.M.; Restante, G.; Gabelloni, M.; Guglielmi, G.; Neri, E. Artificial intelligence, radiomics and other horizons in body composition assessment. Quant. Imaging Med. Surg. 2020, 10, 1650-1660. [CrossRef]

12. Van Timmeren, J.E.; Cester, D.; Tanadini-Lang, S.; Alkadhi, H.; Baessler, B. Radiomics in medical imaging_"How-to" guide and critical reflection. Insights Imaging 2020, 11. [CrossRef]

13. Defeudis, A.; De Mattia, C.; Rizzetto, F.; Calderoni, F.; Mazzetti, S.; Torresin, A.; Vanzulli, A.; Regge, D.; Giannini, V. Standardization of CT radiomics features for multi-center analysis: Impact of software settings and parameters. Phys. Med. Biol. 2020, 65. [CrossRef] 
14. Shafiq-ul-Hassan, M.; Zhang, G.G.; Latifi, K.; Ullah, G.; Hunt, D.C.; Balagurunathan, Y.; Abdalah, M.A.; Schabath, M.B.; Goldgof, D.G.; Mackin, D.; et al. Intrinsic dependencies of CT radiomic features on voxel size and number of gray levels. Med. Phys. 2017, 44, 1050-1062. [CrossRef] [PubMed]

15. Berenguer, R.; Pastor-Juan, M.D.R.; Canales-Vázquez, J.; Castro-García, M.; Villas, M.V.; Mansilla Legorburo, F.; Sabater, S. Radiomics of CT features may be nonreproducible and redundant: Influence of CT acquisition parameters. Radiology 2018, 288, 407-415. [CrossRef]

16. Lv, W.; Yuan, Q.; Wang, Q.; Ma, J.; Jiang, J.; Yang, W.; Feng, Q.; Chen, W.; Rahmim, A.; Lu, L. Robustness versus disease differentiation when varying parameter settings in radiomics features: Application to nasopharyngeal PET/CT. Eur. Radiol. 2018, 28, 3245-3254. [CrossRef]

17. Crombé, A.; Kind, M.; Fadli, D.; Le Loarer, F.; Italiano, A.; Buy, X.; Saut, O. Intensity harmonization techniques influence radiomics features and radiomics-based predictions in sarcoma patients. Sci. Rep. 2020, 10. [CrossRef]

18. Haarburger, C.; Müller-Franzes, G.; Weninger, L.; Kuhl, C.; Truhn, D.; Merhof, D. Radiomics feature reproducibility under inter-rater variability in segmentations of CT images. Sci. Rep. 2020, 10. [CrossRef]

19. Soomro, M.H.; Coppotelli, M.; Conforto, S.; Schmid, M.; Giunta, G.; Del Secco, L.; Neri, E.; Caruso, D.; Rengo, M.; Laghi, A. Automated segmentation of colorectal tumor in 3D MRI using 3D multiscale densely connected convolutional neural network. J. Healthc. Eng. 2019, 2019. [CrossRef]

20. Lambin, P.; Rios-Velazquez, E.; Leijenaar, R.; Carvalho, S.; van Stiphout, R.G.; Granton, P.; Zegers, C.M.; Gillies, R.; Boellard, R.; Dekker, A.; et al. Radiomics: Extracting more information from medical images using advanced feature analysis. Eur. J. Cancer. 2012, 48, 441-446. [CrossRef]

21. Sofka, M.; Wetzl, J.; Birkbeck, N.; Zhang, J.; Kohlberger, T.; Kaftan, J.; Declerck, J.; Zhou, S.K. Multi-stage learning for robust lung segmentation in challenging CT volumes. Med. Image Comput. Comput. Assist. Interv. 2011, 14, 667-674. [CrossRef]

22. Kim, H.; Park, C.M.; Lee, M.; Park, S.J.; Song, Y.S.; Lee, J.H.; Hwang, E.J.; Goo, J.M. Impact of reconstruction algorithms on $\mathrm{CT}$ radiomic features of pulmonary tumors: Analysis of intra-and inter-reader variability and inter-reconstruction algorithm variability. PLOS ONE 2016, 11, e0164924. [CrossRef]

23. Heye, T.; Merkle, E.M.; Reiner, C.S.; Davenport, M.S.; Horvath, J.J.; Feuerlein, S.; Breault, S.R.; Gall, P.; Bashir, M.R.; Dale, B.M.; et al. Reproducibility of dynamic contrast-enhanced MR imaging. Part II. Comparison of intra-and interobserver variability with manual region of interest placement versus semiautomatic lesion segmentation and histogram analysis. Radiology 2013, 266, 812-821. [CrossRef]

24. Rizzetto, F.; Calderoni, F.; De Mattia, C.; Defeudis, A.; Giannini, V.; Mazzetti, S.; Vassallo, L.; Ghezzi, S.; Sartore-Bianchi, A.; Marsoni, S.; et al. Impact of inter-reader contouring variability on textural radiomics of colorectal liver metastases. Eur. Radiol. Exp. 2020, 4. [CrossRef]

25. Zwanenburg, A.; Leger, S.; Vallières, M.; Löck, S. Image biomarker standardisation initiative. arXiv 2019, arXiv:1612.07003.

26. Koçak, B.; Durmaz, E.; Ateş, E.; Kılıçkesmez, Ö. Radiomics with artificial intelligence: A practical guide for beginners. Diagn. Interv. Radiol. 2019, 25, 485-495. [CrossRef]

27. Hatt, M.; Parmar, C.; Qi, J.; Naqa, I.E. Machine (deep) learning methods for image processing and radiomics. IEEE Trans. Radiat. Plasma Med. Sci. 2019, 3. [CrossRef]

28. Varma, S.; Simon, R. Bias in error estimation when using cross-validation for model selection. BMC Bioinform. 2006, 7. [CrossRef]

29. Wu, L.; Wang, C.; Tan, X.; Cheng, Z.; Zhao, K.; Yan, L.; Liang, Y.; Liu, Z.; Liang, C. Radiomics approach for preoperative identification of stages I. Chin. J. Cancer Res. 2018, 30, 396-405. [CrossRef]

30. Liu, S.; Zheng, H.; Pan, X.; Chen, L.; Shi, M.; Guan, Y.; Ge, Y.; He, J.; Zhou, Z. Texture analysis of CT imaging for assessment of esophageal squamous cancer aggressiveness. J. Thorac. Dis. 2017, 9, 4724-4732. [CrossRef]

31. Jin, X.; Zheng, X.; Chen, D.; Jin, J.; Zhu, G.; Deng, X.; Han, C.; Gong, C.; Zhou, Y.; Liu, C.; et al. Prediction of response after chemoradiation for esophageal cancer using a combination of dosimetry and CT radiomics. Eur. Radiol. 2019, 29, 6080-6088. [CrossRef] [PubMed]

32. Hu, Y.; Xie, C.; Yang, H.; Ho, J.W.K.; Wen, J.; Han, L.; Chiu, K.W.H.; Fu, J.; Vardhanabhuti, V. Assessment of intratumoral and peritumoral computed tomography radiomics for predicting pathological complete response to neoadjuvant chemoradiation in patients with esophageal squamous cell carcinoma. JAMA Netw. Open 2020, 3, e2015927. [CrossRef]

33. Nakajo, M.; Jinguji, M.; Nakabeppu, Y.; Higashi, R.; Fukukura, Y.; Sasaki, K.; Uchikado, Y.; Natsugoe, S.; Yoshiura, T. Texture analysis of 18 F-FDG PET/CT to predict tumour response and prognosis of patients with esophageal cancer treated by chemoradiotherapy. Eur. J. Nucl. Med. Mol. Imaging 2017, 44, 206-214. [CrossRef]

34. Wu, L.; Yang, X.; Cao, W.; Zhao, K.; Li, W.; Ye, W.; Chen, X.; Zhou, Z.; Liu, Z.; Liang, C. Multiple level CT Radiomics features preoperatively predict lymph node metastasis in esophageal cancer: A multicentre retrospective study. Front. Oncol. 2019, 9. [CrossRef]

35. Shen, C.; Liu, Z.; Wang, Z.; Guo, J.; Zhang, H.; Wang, Y.; Qin, J.; Li, H.; Fang, M.; Tang, Z.; et al. Building CT radiomics based nomogram for preoperative esophageal cancer patients lymph node metastasis prediction. Transl. Oncol. 2018, 11, 815-824. [CrossRef] [PubMed]

36. Qiu, Q.; Duan, J.; Deng, H.; Han, Z.; Gu, J.; Yue, N.J.; Yin, Y. Development and Validation of a radiomics nomogram model for predicting postoperative recurrence in patients with esophageal squamous cell cancer who achieved pCR after neoadjuvant chemoradiotherapy followed by surgery. Front. Oncol. 2020, 10. [CrossRef] 
37. Liu, S.; Ji, C.; Zheng, H.; Pan, X.; Zhang, Y.; Guan, W.; Chen, L.; Guan, Y.; Li, W.; He, J.; et al. Application of CT texture analysis in predicting histopathological characteristics of gastric cancers. Eur. Radiol. 2017, 27, 4951-4959. [CrossRef]

38. Wang, Y.; Liu, W.; Yu, Y.; Liu, J.J.; Xue, H.D.; Qi, Y.F.; Lei, J.; Yu, J.C.; Jin, Z.Y. CT radiomics nomogram for the preoperative prediction of lymph node metastasis in gastric cancer. Eur. Radiol. 2020, 30, 976-986. [CrossRef]

39. Dong, D.; Tang, L.; Li, Z.Y.; Fang, M.J.; Gao, J.B.; Shan, X.H.; Ying, X.J.; Sun, Y.S.; Fu, J.; Wang, X.X.; et al. Development and validation of an individualized nomogram to identify occult peritoneal metastasis in patients with advanced gastric cancer. Ann. Oncol. 2019, 30, 431-438. [CrossRef]

40. Giganti, F.; Antunes, S.; Salerno, A.; Ambrosi, A.; Marra, P.; Nicoletti, R.; Orsenigo, E.; Chiari, D.; Albarello, L.; Staudacher, C.; et al. Gastric cancer: Texture analysis from multidetector computed tomography as a potential preoperative prognostic biomarker. Eur. Radiol. 2017, 27, 1831-1839. [CrossRef]

41. Li, W.; Zhang, L.; Tian, C.; Song, H.; Fang, M.; Hu, C.; Zang, Y.; Cao, Y.; Dai, S.; Wang, F.; et al. Prognostic value of computed tomography radiomics features in patients with gastric cancer following curative resection. Eur. Radiol. 2019, 29, 3079-3089. [CrossRef] [PubMed]

42. Jiang, Y.; Chen, C.; Xie, J.; Wang, W.; Zha, X.; Lv, W.; Chen, H.; Hu, Y.; Li, T.; Yu, J.; et al. Radiomics signature of computed tomography imaging for prediction of survival and chemotherapeutic benefits in gastric cancer. EBioMedicine 2018, 36, 171-182. [CrossRef] [PubMed]

43. Sun, K.Y.; Hu, H.T.; Chen, S.L.; Ye, J.N.; Li, G.H.; Chen, L.D.; Peng, J.J.; Feng, S.T.; Yuan, Y.J.; Hou, X.; et al. CT-based radiomics scores predict response to neoadjuvant chemotherapy and survival in patients with gastric cancer. BMC Cancer 2020, 20. [CrossRef] [PubMed]

44. Mokrane, F.Z.; Lu, L.; Vavasseur, A.; Otal, P.; Peron, J.M.; Luk, L.; Yang, H.; Ammari, S.; Saenger, Y.; Rousseau, H.; et al. Radiomics machine-learning signature for diagnosis of hepatocellular carcinoma in cirrhotic patients with indeterminate liver nodules. Eur. Radiol. 2020, 30, 558-570. [CrossRef]

45. Wu, M.; Tan, H.; Gao, F.; Hai, J.; Ning, P.; Chen, J.; Zhu, S.; Wang, M.; Dou, S.; Shi, D. Predicting the grade of hepatocellular carcinoma based on non-contrast-enhanced MRI radiomics signature. Eur. Radiol. 2019, 29, 2802-2811. [CrossRef]

46. Yang, C.; Huang, M.; Li, S.; Chen, J.; Yang, Y.; Qin, N.; Huang, D.; Shu, J. Radiomics model of magnetic resonance imaging for predicting pathological grading and lymph node metastases of extrahepatic cholangiocarcinoma. Cancer Lett. 2020, 470, 1-7. [CrossRef]

47. Kim, J.; Choi, S.J.; Lee, S.H.; Lee, H.Y.; Park, H. Predicting survival using pretreatment CT for patients with hepatocellular carcinoma treated with transarterial chemoembolization: Comparison of models using radiomics. AJR Am. J. Roentgenol. 2018, 211, 1026-1034. [CrossRef]

48. Min, J.H.; Lee, M.W.; Park, H.S.; Lee, D.H.; Park, H.J.; Lim, S.; Choi, S.Y.; Lee, J.; Lee, J.E.; Ha, S.Y.; et al. Interobserver variability and diagnostic performance of gadoxetic acid-enhanced MRI for predicting microvascular invasion in hepatocellular carcinoma. Radiology 2020, 297, 573-581. [CrossRef] [PubMed]

49. Xu, X.; Zhang, H.L.; Liu, Q.P.; Sun, S.W.; Zhang, J.; Zhu, F.P.; Yang, G.; Yan, X.; Zhang, Y.D.; Liu, X.S. Radiomic analysis of contrast-enhanced CT predicts microvascular invasion and outcome in hepatocellular carcinoma. J. Hepatol. 2019, 70, 1133-1144. [CrossRef] [PubMed]

50. Ni, M.; Zhou, X.; Lv, Q.; Li, Z.; Gao, Y.; Tan, Y.; Liu, J.; Liu, F.; Yu, H.; Jiao, L.; et al. Radiomics models for diagnosing microvascular invasion in hepatocellular carcinoma: Which model is the best model? Cancer Imaging 2019, 19. [CrossRef]

51. Feng, S.T.; Jia, Y.; Liao, B.; Huang, B.; Zhou, Q.; Li, X.; Wei, K.; Chen, L.; Li, B.; Wang, W.; et al. Preoperative prediction of microvascular invasion in hepatocellular cancer: A radiomics model using Gd-EOB-DTPA-enhanced MRI. Eur. Radiol. 2019, 29, 4648-4659. [CrossRef]

52. Ji, G.W.; Zhu, F.P.; Xu, Q.; Wang, K.; Wu, M.Y.; Tang, W.W.; Li, X.C.; Wang, X.H. Machine-learning analysis of contrast-enhanced $\mathrm{CT}$ radiomics predicts recurrence of hepatocellular carcinoma after resection: A multi-institutional study. EBioMedicine 2019, 50, 156-165. [CrossRef]

53. Liang, W.; Xu, L.; Yang, P.; Zhang, L.; Wan, D.; Huang, Q.; Niu, T.; Chen, F. Novel Nomogram for preoperative prediction of early recurrence in intrahepatic cholangiocarcinoma. Front. Oncol. 2018, 8. [CrossRef] [PubMed]

54. Taghavi, M.; Trebeschi, S.; Simões, R.; Meek, D.B.; Beckers, R.C.J.; Lambregts, D.M.J.; Verhoef, C.; Houwers, J.B.; van der Heide, U.A.; Beets-Tan, R.G.H.; et al. Machine learning-based analysis of CT radiomics model for prediction of colorectal metachronous liver metastases. Abdom. Radiol. 2020. [CrossRef] [PubMed]

55. Ren, S.; Zhao, R.; Zhang, J.; Guo, K.; Gu, X.; Duan, S.; Wang, Z.; Chen, R. Diagnostic accuracy of unenhanced CT texture analysis to differentiate mass-forming pancreatitis from pancreatic ductal adenocarcinoma. Abdom. Radiol. 2020, 45, 1524-1533. [CrossRef] [PubMed]

56. Chu, L.C.; Park, S.; Kawamoto, S.; Fouladi, D.F.; Shayesteh, S.; Zinreich, E.S.; Graves, J.S.; Horton, K.M.; Hruban, R.H.; Yuille, A.L.; et al. Utility of CT radiomics features in differentiation of pancreatic ductal adenocarcinoma from normal pancreatic tissue. AJR Am. J. Roentgenol. 2019, 213, 349-357. [CrossRef]

57. Park, S.; Chu, L.C.; Hruban, R.H.; Vogelstein, B.; Kinzler, K.W.; Yuille, A.L.; Fouladi, D.F.; Shayesteh, S.; Ghandili, S.; Wolfgang, C.L.; et al. Differentiating autoimmune pancreatitis from pancreatic ductal adenocarcinoma with CT radiomics features. Diagn. Interv. Imaging 2020, 101, 555-564. [CrossRef] 
58. Tobaly, D.; Santinha, J.; Sartoris, R.; Dioguardi Burgio, M.; Matos, C.; Cros, J.; Couvelard, A.; Rebours, V.; Sauvanet, A.; Ronot, M.; et al. CT-based radiomics analysis to predict malignancy in patients with intraductal papillary mucinous neoplasm (IPMN) of the pancreas. Cancers 2020, 12, 3089. [CrossRef]

59. Nasief, H.; Zheng, C.; Schott, D.; Hall, W.; Tsai, S.; Erickson, B.; Allen Li, X. A machine learning based delta-radiomics process for early prediction of treatment response of pancreatic cancer. NPJ Precis. Oncol. 2019, 3. [CrossRef]

60. Parr, E.; Du, Q.; Zhang, C.; Lin, C.; Kamal, A.; McAlister, J.; Liang, X.; Bavitz, K.; Rux, G.; Hollingsworth, M.; et al. Radiomics-based outcome prediction for pancreatic cancer following stereotactic body radiotherapy. Cancers 2020, 12, 1051. [CrossRef]

61. Eilaghi, A.; Baig, S.; Zhang, Y.; Zhang, J.; Karanicolas, P.; Gallinger, S.; Khalvati, F.; Haider, M.A. CT texture features are associated with overall survival in pancreatic ductal adenocarcinoma-A quantitative analysis. BMC Med. Imaging 2017, 17. [CrossRef] [PubMed]

62. Kim, B.R.; Kim, J.H.; Ahn, S.J.; Joo, I.; Choi, S.Y.; Park, S.J.; Han, J.K. CT prediction of resectability and prognosis in patients with pancreatic ductal adenocarcinoma after neoadjuvant treatment using image findings and texture analysis. Eur. Radiol. 2019, 29, 362-372. [CrossRef] [PubMed]

63. Tang, T.Y.; Li, X.; Zhang, Q.; Guo, C.X.; Zhang, X.Z.; Lao, M.Y.; Shen, Y.N.; Xiao, W.B.; Ying, S.H.; Sun, K.; et al. Development of a novel multiparametric MRI radiomic nomogram for preoperative evaluation of early recurrence in resectable pancreatic cancer. J. Magn. Reson. Imaging 2020, 52, 231-245. [CrossRef]

64. Benson, A.B.; Venook, A.P.; Al-Hawary, M.M.; Arain, M.A.; Chen, Y.J.; Ciombor, K.K.; Cohen, S.A.; Cooper, H.S.; Deming, D.A.; Garrido-Laguna, I.; et al. Small bowel adenocarcinoma, version 1.2020, NCCN clinical practice guidelines in oncology. J. Natl. Compr. Cancer Netw. 2019, 17, 1109-1133. [CrossRef] [PubMed]

65. Lu, J.; Hu, D.; Tang, H.; Hu, X.; Shen, Y.; Li, Z.; Peng, Y.; Kamel, I. Assessment of tumor heterogeneity: Differentiation of periampullary neoplasms based on CT whole-lesion histogram analysis. Eur. J. Radiol. 2019, 115, 1-9. [CrossRef]

66. Ren, C.; Wang, S.; Zhang, S. Development and validation of a nomogram based on CT images and 3D texture analysis for preoperative prediction of the malignant potential in gastrointestinal stromal tumors. Cancer Imaging 2020, 20. [CrossRef]

67. Wang, C.; Li, H.; Jiaerken, Y.; Huang, P.; Sun, L.; Dong, F.; Huang, Y.; Dong, D.; Tian, J.; Zhang, M. Building CT radiomics-based models for preoperatively predicting malignant potential and mitotic count of gastrointestinal stromal tumors. Transl. Oncol. 2019, 12, 1229-1236. [CrossRef]

68. Zhang, Q.W.; Gao, Y.J.; Zhang, R.Y.; Zhou, X.X.; Chen, S.L.; Zhang, Y.; Liu, Q.; Xu, J.R.; Ge, Z.Z. Personalized CT-based radiomics nomogram preoperative predicting Ki-67 expression in gastrointestinal stromal tumors: A multicenter development and validation cohort. Clin. Transl. Med. 2020, 9. [CrossRef]

69. Chen, T.; Liu, S.; Li, Y.; Feng, X.; Xiong, W.; Zhao, X.; Yang, Y.; Zhang, C.; Hu, Y.; Chen, H.; et al. Developed and validated a prognostic nomogram for recurrence-free survival after complete surgical resection of local primary gastrointestinal stromal tumors based on deep learning. EBioMedicine 2019, 39, 272-279. [CrossRef]

70. Ekert, K.; Hinterleitner, C.; Horger, M. Prognosis assessment in metastatic gastrointestinal stromal tumors treated with tyrosine kinase inhibitors based on CT-texture analysis. Eur. J. Radiol. 2019, 116, 98-105. [CrossRef]

71. Guo, C.; Zhuge, X.; Wang, Z.; Wang, Q.; Sun, K.; Feng, Z.; Chen, X. Textural analysis on contrast-enhanced CT in pancreatic neuroendocrine neoplasms: Association with WHO grade. Abdom. Radiol. 2019, 44, 576-585. [CrossRef]

72. Gu, D.; Hu, Y.; Ding, H.; Wei, J.; Chen, K.; Liu, H.; Zeng, M.; Tian, J. CT radiomics may predict the grade of pancreatic neuroendocrine tumors: A multicenter study. Eur. Radiol. 2019, 29, 6880-6890. [CrossRef] [PubMed]

73. Bian, Y.; Zhao, Z.; Jiang, H.; Fang, X.; Li, J.; Cao, K.; Ma, C.; Guo, S.; Wang, L.; Jin, G.; et al. Noncontrast radiomics approach for predicting grades of nonfunctional pancreatic neuroendocrine tumors. J. Magn. Reson. Imaging 2020, 52, 1124-1136. [CrossRef] [PubMed]

74. He, M.; Liu, Z.; Lin, Y.; Wan, J.; Li, J.; Xu, K.; Wang, Y.; Jin, Z.; Tian, J.; Xue, H. Differentiation of atypical non-functional pancreatic neuroendocrine tumor and pancreatic ductal adenocarcinoma using CT based radiomics. Eur. J. Radiol. 2019, 117, 102-111. [CrossRef] [PubMed]

75. Martini, I.; Polici, M.; Zerunian, M.; Panzuto, F.; Rinzivillo, M.; Landolfi, F.; Magi, L.; Caruso, D.; Eid, M.; Annibale, B.; et al. CT texture analysis of liver metastases in PNETs versus NPNETs: Correlation with histopathological findings. Eur. J. Radiol. 2020, 124. [CrossRef]

76. Caruso, D.; Zerunian, M.; Ciolina, M.; de Santis, D.; Rengo, M.; Soomro, M.H.; Giunta, G.; Conforto, S.; Schmid, M.; Neri, E.; et al. Haralick's texture features for the prediction of response to therapy in colorectal cancer: A preliminary study. Radiol. Med. 2018, 123, 161-167. [CrossRef]

77. De Cecco, C.N.; Ciolina, M.; Caruso, D.; Rengo, M.; Ganeshan, B.; Meinel, F.G.; Musio, D.; De Felice, F.; Tombolini, V.; Laghi, A. Performance of diffusion-weighted imaging, perfusion imaging, and texture analysis in predicting tumoral response to neoadjuvant chemoradiotherapy in rectal cancer patients studied with 3T MR: Initial experience. Abdom. Radiol. 2016, 41, 1728-1735. [CrossRef]

78. Badic, B.; Tixier, F.; Cheze Le Rest, C.; Hatt, M.; Visvikis, D. Radiogenomics in colorectal cancer. Cancers 2021, 13, 973. [CrossRef]

79. Granata, V.; Fusco, R.; Avallone, A.; De Stefano, A.; Ottaiano, A.; Sbordone, C.; Brunese, L.; Izzo, F.; Petrillo, A. Radiomicsderived data by contrast enhanced magnetic resonance in RAS mutations detection in colorectal liver metastases. Cancers 2021, 13, 453. [CrossRef] 
80. Alvarez-Jimenez, C.; Antunes, J.T.; Talasila, N.; Bera, K.; Brady, J.T.; Gollamudi, J.; Marderstein, E.; Kalady, M.F.; Purysko, A.; Willis, J.E.; et al. Radiomic texture and shape descriptors of the rectal environment on post-chemoradiation T2-Weighted MRI are associated with pathologic tumor stage regression in rectal cancers: A retrospective, multi-institution study. Cancers 2020, 12, 2027. [CrossRef]

81. Hamerla, G.; Meyer, H.J.; Hambsch, P.; Wolf, U.; Kuhnt, T.; Hoffmann, K.T.; Surov, A. Radiomics model based on non-contrast CT Shows no predictive power for complete pathological response in locally advanced rectal cancer. Cancers 2019, 11, 1680. [CrossRef] [PubMed]

82. Yang, L.; Dong, D.; Fang, M.; Zhu, Y.; Zang, Y.; Liu, Z.; Zhang, H.; Ying, J.; Zhao, X.; Tian, J. Can CT-based radiomics signature predict KRAS/NRAS/BRAF mutations in colorectal cancer? Eur. Radiol. 2018, 28, 2058-2067. [CrossRef] [PubMed]

83. Taguchi, N.; Oda, S.; Yokota, Y.; Yamamura, S.; Imuta, M.; Tsuchigame, T.; Nagayama, Y.; Kidoh, M.; Nakaura, T.; Shiraishi, S.; et al. CT texture analysis for the prediction of KRAS mutation status in colorectal cancer via a machine learning approach. Eur. J. Radiol. 2019, 118, 38-43. [CrossRef]

84. Golia Pernicka, J.S.; Gagniere, J.; Chakraborty, J.; Yamashita, R.; Nardo, L.; Creasy, J.M.; Petkovska, I.; Do, R.R.K.; Bates, D.D.B.; Paroder, V.; et al. Radiomics-based prediction of microsatellite instability in colorectal cancer at initial computed tomography evaluation. Abdom. Radiol. 2019, 44,3755-3763. [CrossRef] [PubMed]

85. Li, M.; Zhang, J.; Dan, Y.; Yao, Y.; Dai, W.; Cai, G.; Yang, G.; Tong, T. A clinical-radiomics nomogram for the preoperative prediction of lymph node metastasis in colorectal cancer. J. Transl. Med. 2020, 18. [CrossRef] [PubMed]

86. Song, L.; Yin, J. Application of texture analysis based on sagittal fat-suppression and oblique Axial T2-weighted magnetic resonance imaging to identify lymph node invasion status of rectal cancer. Front. Oncol. 2020, 10. [CrossRef]

87. Yang, L.; Liu, D.; Fang, X.; Wang, Z.; Xing, Y.; Ma, L.; Wu, B. Rectal cancer: Can T2WI histogram of the primary tumor help predict the existence of lymph node metastasis? Eur. Radiol. 2019, 29, 6469-6476. [CrossRef]

88. Horvat, N.; Veeraraghavan, H.; Khan, M.; Blazic, I.; Zheng, J.; Capanu, M.; Sala, E.; Garcia-Aguilar, J.; Gollub, M.J.; Petkovska, I. MR imaging of rectal cancer: Radiomics analysis to assess treatment response after neoadjuvant therapy. Radiology 2018, 287, 833-843. [CrossRef] 